\title{
Lieber Freund!
}

Am 3. I1. 46 erhielt ich Deinen Brief vom 14.Juli d.Jrs. und freue mich sehr darüber, $\mathrm{daß}$ Ihr uns da druben nicht vergessen habt. Gleichzeitig danke ich Dir für Deine Glückwünsche, daß ich trotz aller Bedrängnis noch so davongekommen bin. Allerdings mit der Einschränkung, daß ich zu 70\% körperbehindert bin, aber dies und alle damit verbundenen körperlichen Schmerzen hindern mich nicht, wieder meinen Mann zu stehen im Kampf der Klasse gegen Klasse.

Ihr glaubt nicht, welche Verwuistungen die Nazis hier in den Köpfen der Menschen durch ihren geistigen Terror angerichtet haben, deshalb hielt ich es für meine Pflicht, am Wiederautbau der Arbeiterbewegung schon aus diesem Grunde teilzunehmen. In ihr sind, im Gegensatz zu den bürgerlichen Schichten, vor allem den Intellektuellen, deren geistiger Stolz vor den Nazis schnell vor die Hunde ging, relativ noch die geistig klarsten Gedanken vertreten. Wie sich die politischen Geister scheiden, werdet Ihr durch die Ausgänge der Wahlen in den verschiedenen Zonen festgestellt haben. Was niemand für möglich gehalten hätte nach diesem fürchterlichen Regime ist, daß die Arbeiterschaft vom Kommunismus, genauer gesagt von der Sowjetunion aufs schwerste enttäuscht wurde und noch ständig weiter wird. So wird die SPD in immer erhöhtem Maße wieder die Partei, der die Massen zuströmen. Die SPD von heute ist auch nicht mehr die Partei von vor 1933, auch sie hat eine gewaltige Lehre aus den Dingen gezogen und zieht sie noch. Dies zeigte auch die Landeskonferenz der Bayerischen SPD in Ingolstadt vor drei Wochen, an der ich als Delegierter von Bamberg teilnahm. Auf der Konferenz herrschte ein erfreulicher frischer oppositioneller Wind, der Hoegner beinahe seine Stellung als Vorsitzender kostete'. Auf der Konferenz traf ich auch eine Reihe ehemaliger SAP-Genossen, die vor allem in München, Schwaben und Oberfranken eine Rolle spielen. Auch die jungsozialistische Bewegung rührt sich immer kräftiger, nur fehlt es uns allen an geistigem Werkzeug. Leider hat auch mir die Gestapo meine herrliche und mühevoll aufgebaute Bibliothek gestohlen. So macht mir meine Tätigkeit als Referent in der Partei, den Jungsozialisten und an der Volkshochschule manchmal Sorge.

Wenn Ihr Eure Aktivität nunmehr auf das Gebiet der Linderung unserer materiellen Nöte legt, so ist Euch das hoch anzurechnen und zeigt uns, daß die sozialistische Treue kein leerer Wahn ist. Dafür danke ich Euch schon im Voraus. Die Bekleidung bei uns

\footnotetext{
'Am 19./20. 10. 1946 fand in Ingolstadt die zweite Landeskonferenz der bayerischen SPD statt, in deren Mittelpunkt die Verabschiedung der vornehmlich vom SPD-Landesvorsizzenden und Ministerpräsidenten Wilhelm Hoegner (1887-1980) gefertigten Satzung für den SPD-Landesverband stand. Dabei zeigte sich bereits Opposition gegen Hoegners stark föderalistische, auf betonte Eigenständigkeit des bayerischen SPD-Landesverbandes hinauslaufende organisatorische und politische Vorstellungen, die denen des SPD-Parteivorstandes unter Kurt Schumacher in Hannover zunehmend entgegenliefen. Diese Opposition nahm in den Folgemonaten stark zu und trug mit dazu bei, daß Hoegner Anfang 1947 als Landesvorsitzender zurücktrat.
} 
ist auch ein Kapitel für sich. Was mich anbelangt, so habe ich im Konzentrationslager alles an Wäsche und Bekleidung eingebüßt und bin von Dachau aus in einem Drillichanzug und Filzstiefeln entlassen worden. Inzwischen habe ich aus einer Kleidersammlung, zu der ehemalige Pgs natürlich ihre allerschlechtesten Anzüge abliefern mußten, auch einen erhalten, das ist alles. Mein ganzes übriges $\mathrm{Hab}$ und Gut haben wir bei der Zwangsevakuierung aus Schlesien restlos verloren. Natürlich würde ich mich sehr freuen, wenn Ihr mir an Kleidungsstücken etwas aushelfen könntet. Größe $1,65 \mathrm{~m}$, Brustumfang $0,95 \mathrm{~m}$, Hosenlänge insgesamt $1,00 \mathrm{~m}$. Hoffentlich genügen Dir diese Größenangaben. Meine Frau und meine beiden Töchter benötigen aufs dringendste Strümpfe für mittlere Größen. Wenn Du an Medikamenten etwas auftreiben könntest, so wäre ich Dir dankbar für Perubalsam, Jod und einige Mullbinden.

So, das wäre mein Wunschzettel, wie Du ihn erwünschtest.

Zur Zeit stehen wir mitten im Wahlkampf und hoffen, den Einfluß der CSU noch mehr zurückzudrängen, zumal die Stimmen der Flüchtlinge in hohem Maße für uns in die Waagschale fallen werden. Je mehr die CSU sich christlich nennt, um so reaktionärere Zielsetzungen hat sie. Nach ihren neuen Kandidaten zu beurteilen, bemüht sie sich den starken linken Flügel der verfassungsgebenden Landesversammlung auszumerzen und das geistliche Element in ihren Kandidatenlisten zu verstärken. Uns soll es recht sein, je klarer die Fronten, um so schärfer scheiden sich die Geister. Große Schwierigkeiten stehen unserem wirtschaftlichen Aufbau bevor. Der Rohstoffmangel ist das Schlimmste. Wenn auch das Großkapital äußerlich zerschlagen da steht, sind wir uns darüber klar, daß es sich wieder konsolidieren wird. Von einer Bedrohung der Weltsicherheit kann nur der reden, der bei uns irgend ein Geschäft wittert, und bei einer Auseinandersetzung zwischen Ost und West zöge der Osten ohne weiteres den kürzeren.

Nun lieber Freund hoffe ich, daß Dich und alle lieben Freunde dieser Brief bei bester Gesundheit antreffen möge, es wird nicht der letzte sein und ich möchte nur wünschen, daß dieser Meinungsaustausch, den ich gern fortsetzen möchte, für uns alle von Interesse sein wird.

Es grüßt Dich und alle Freunde herzlichst

Euer Fritz Nagel

Fritz Nagel an Joseph und Ema Lang

Bamberg, den 5.2. 1947

Memmelsdorferstr. 2a

Mein lieber Freund Jola und Erna!

Gerade am letzten Tage des alten Jahres traf Dein Brief vom I s. I 2. ein und löste keine geringe Freude aus. Und vor einigen Tagen erhielt ich erst ein Paket von Dir mit einem Anzug, Unterwäsche, Strümpfe, Medikamenten und diversen Vitamintabletten. Bald darauf folgte das andere mit dem Kleid, Schuhen und Lebensmitteln, das ich mir aber von Nürnberg abholen lassen mußte, wo es schon seit November bei Freunden lag, denen meine Adresse nicht sofort bekannt war. Alles war in bestem Zustand und unsere Freude groß, zumal an derartige Dinge hier nicht zu denken ist. Deine und aller anderen Freunde Fürsorge für mein Wohl ist doch sehr anerkennenswert, nur weiß ich nicht, wie ich Euch dafür danken soll. Konrad Reisner* hat mir aus Minneapolis einen Brief geschrieben, aus dem hervorgeht, daß er sich im gleichen Sinne bemüht. Selbst- 
verständlich werde ich über die Verwendung der Vitamine mit einem Arzt in Verbindung treten. $\mathrm{Zu}$ meinem Leiden kann ich Dir nur sagen, daß der rechte Schambeinknochen durch eine aus der Unfallstelle hervorgegangene Wucherung stark zerstört ist und ich, abgesehen von meiner Körperbehinderung beim Gehen, unter erheblichen Schmerzen leide, vor allem des nachts. Ohne schmerzlindernde Mittel (Tabletten) komme ich leider nicht aus. Ein Kriegsbeschädigter, der ein Bein verloren hat, ist darin besser dran, er hat wenigstens keine Schmerzen. Der Arzt hat mir verordnet, viel zu liegen, um den Beckenknochen zu schonen. Geistige Arbeit während der Nacht hilft mir, denn das Schlafen habe ich mir fast abgewöhnt, und Schlafmittel möchte ich so wenig wie möglich anwenden. Um das weitere $W$ achstum der Wucherung zu verhindern (die Krankheit wurde ursprünglich ostitis fibrosa genannt), erhielt ich im vorigen Jahr vier Röntgenbestrahlungen in der Univ. Klinik Erlangen, deren Auswirkungen allerdings Zeit gebraucht. Nie wäre es so weit gekommen, wenn im KZ-Lager eine Behandlung moglich gewesen wäre. Ihr werdet aus diesen Folterhöhlen des Dritten Reiches manches Bild gesehen und manche Schilderung gelesen haben, doch die Wirklichkeit ist niemals wiederzugeben. So ist es logisch, daß wir Antifaschisten, die durch diese Höllen gegangen sind, die sogenannte Entnazifizierung mit Argusaugen betrachten. Hier haben wir auch ein Vermächtnis unserer durch die Nazis zugrunde gerichteten Freunde zu erfüllen. Bilder wie das unseres lieben Ernst Eckstein* und vieler anderer stehen mir immer vor Augen so lange ich leben werde. Freilich, der Geist Hitler spukt in Deutschland noch genug herum, gefördert durch die schlechte ökonomische Lage, vor allem in der Jugend, welche noch nichts anderes kennengelernt hat. $\mathrm{Da}_{\mathrm{a}}$ aber diese Ideenwelt noch einmal ihre Auferstehung feiert, ist ein Unding. Der Nationalismus hat hier in Europa Orgien zur Genüge gefeiert und feiert sie leider noch. Karl Renner hat durchaus recht wenn er meint, daß die Auflösung des Habsburger Reiches, in dem es gar nicht einmal so untolerant zuging, seinen Völkern kein Gluick gebracht hat', wohl aber, wie ich dazu meine, einer ganzen Reihe von Leuten einträgliche Ministersessel und der Rüstungsindustrie große Aufträge. Die Freiheit der Volker ist nur eine suggestive Hypothese geblieben. Wie viele Völker leben nicht friedlich in den U.S.A. zusammen, aber in Europa werden die Minderheitenfragen so gelöst, daß dabei noch mehr Minderheiten geschaffen werden und sich am Ende einer vor lauter Nationalitäten nicht mehr auskennt. Mit solchem Theater werden nur die Geister abgelenkt von der großen Frage über unser Kapitalverhältnis. Auch in Deutschland hört man viel von Föderalismus, von der beabsichtigten Bildung von so und so viel Staaten, mit Parlamenten, Regierungen, außenpolitischen Vertretungen und was weiß ich alles. Und andererseits wird wieder viel geredet von Sparmaßnahmen, niedrigerem Lebensstandard, Reparationen u.ä., Widersprüche über Widersprüche. Ich lese häufig in CSU-Blättern, daß der Zentralismus des Weimarer Systems nicht mehr wiederkommen darf, wohl, weil er der CSU nicht genug Pfründe bringt, dabei ist bekannt, daß es der Berliner Regierung vor 1933 nicht einmal möglich war, den Hitler des Landes zu verweisen, weil die Befugnisse der Länder dem im Wege standen und so dieser Bandit schließlich in Braunschweig Regierungsrat werden konnte.

\footnotetext{
' Karl Renner (1870-1950): führender Vertreter der österreichischen Sozialdemokraten, 1918-1920 österreichischer Staatskanzler. Nach dem Februaraufstand 1934 in Haft, 1938 stimmte er dem Anschluß an Deutschland zunächst zu. 1945-1950 osterreichischer Bundespräsident. Seit 1899 veröffentlichte Renner mehrere Bücher und Broschüren zur Nationalitätenfrage, in denen er für eine bundesstaatliche Ordnung des Vielvölkerstaates eintrat.
} 
Unter den politischen Gebilden des heutigen Deutschlands, wenigstens der Westzonen, ist die SPD der ruhende Pol in der Erscheinungen Flucht geworden. Für uns ist das Zerreißen des deutschen Reichsgebildes ein Zerreißen des Wirtschaftskörpers, der gerade im Interesse Europas als Einheit erhalten werden muß. Die Durchführung der Sozialisierung der Schlüsselindustrien, die heute nicht mehr wie 1919 diskutiert wird, sondern in das Stadium ihrer akuten Durchführung getreten ist, bietet auch die beste Garantie für die Sicherheit der Völker. In der Kontrolle der Schwerindustrie durch das Volk liegt diese Bürgschaft begründet. Imperialistischen Geschäftemachern bringt allerdings diese Lösung nichts. Hier winken keine Profite. Mit Dr. Schumacher hat die Partei ohne mit Überschwang zu sagen einen Führer, der in seiner Konsequenz und Popularität seit Bebel nicht vorhanden war, und durch ihn ist sie ein so festes einheitliches Gebilde geworden, wie kein zweites in Deutschland.

Wenn Freund Walcher* herüberkommt, wird er die Dinge in ihrem tatsächlichen Aussehen kennenlernen, die er drüben nur in einer ideologischen Gewünschtheit schimmern sah. Wir haben und können am Osten viel lernen, der uns vor allem zeigt, daß der Ablauf der Geschichte keine konstante Größe ist und der Mensch ein unberechenbares Wesen.

Unter uns deutschen Antifaschisten gab es eine helle Empörung, als wir hörten, daß eine deutsche Frau Dr. Gabriele Strecker auf einem in den U.S.A. abgehaltenen Frauenkongreß erzählte, daß es im Dritten Reich gar keine Widerstandsbewegung gegeben hätte ${ }^{2}$. Die Dame hat ja nie mit Linksparteien in Berührung gestanden, denn sonst hätte sie wissen müssen, wieviel Menschen in den Jahren 1933-1938 in die Gefängnisse, Zuchthäuser und KZ-Lager von uns gewandert sind. 1936 waren es fast 40000 . Da gab es z. B. Massenprozesse wie in Hamm i. W., wo Gruppen bis zu 2500 Mann abgeurteilt wurden ${ }^{3}$. In jeder Einmannzelle lagen wir 3 Gefangene und weil die Anstalten nicht mehr ausreichten, wurden auch für Strafgefangene immer mehr Arbeitslager geschaffen.

Nun einiges über unsere SAP-Freunde ${ }^{4}$ : Max Rausch wohnt in Görlitz und ist Sekretär bei der SED, Willi Kalinke unser letzter Bezirksleiter in der Illegalität lebt wie ich hörte in Erfurt, er war zu 12 Jahren Zuchthaus verurteilt; Fritz Sommer aus Breslau wurde nach s Jahren Zuchthaus in ein Strafbataillon gesteckt und ist bei einem Fliegerangriff auf den Dodekanes-Inseln gefallen. Max Rettig lebt in Berlin, Arnold Trupke in Dresden N. 23 , Wilder Mann-Str. 23 e, er war Distriktleiter in Breslau und ihm geht es ge-

${ }^{2}$ Gabriele Strecker, geb. 1904, Ärztin und Publizistin, 1946-1962 Leiterin des Frauenfunks des Hessischen Rundfunks, 1954-1962 MdL Hessen (CDU), I958-1966 Mitglied des Bundesvorstandes der CDU; wurde als erste deutsche Frau nach dem Ende des Zweiten Weltkrieges zu einer Internationalen Frauenkonferenz eingeladen, die im Oktober 1946 in South Kortright, New York, stattfand. In einem Interview mit der New York Times am I 5. 10. 1946 sagte Gabriele Strecker auf die Frage nach der Beteiligung von Frauen in der deutschen Widerstandsbewegung in Deutschland: „Es gab keine Widerstandsbewegung in Deutschland. Das ist eine Tatsache, und das müssen wir zugeben." $\mathrm{Vgl}$. dazu ihre eigene Darstellung in: Gabriele Strecker: Überleben ist nicht genug. Frauen I945-1950. Freiburg i. Br. 1981, S. 34 f.

3 Diese Angaben konnten nicht verifiziert werden.

4 Die im folgenden Genannten gehörten alle der illegalen SAP in Breslau an. Willi Kalinke und Fritz Sommer wurden Anfang 1936 verhaftet, die Lehrerin Ilse Hacks* und Herbert A. Tulatz* im September 1937. Der Laboratoriumsdiener Max Rettig, der Metallarbeiter Arnold Trupke und der Buchhalter Siegfried Pech wurden im Frühjahr 1937 vom Oberlandesgericht Breslau zu je drei Jahren Zuchthaus verurteilt. Der Schneider Oskar Krummschmidt war von 1939 bis 194 I im KZ. Zu Einzelheiten des SAP-Widerstandes in Breslau vgl. Jörg Bremer: Die Sozialistische Arbeiterpartei Deutschlands (SAP). Untergrund und Exil 1933-45. Frankfurt a. M. 1978, S. 76-80. 
sundheitlich sehr schlecht, Herbert Tulatz ${ }^{*}$, der noch nach unserer Verhaftung mit Ilse $\mathrm{Hacks}^{*}$ in Breslau die Sache weiter leitete, bis auch diese das Schicksal ereilte, lebt in Reutlingen und gibt dort die Jugendzeitschrift „Die Zukunft" mit heraus. Unser früherer SAP-Freund Hans Ziegler*, früher Sekretär des Metallarbeiterverbandes in Breslau, ist seit 1945 Oberbürgermeister in Nürnberg. Mit einer ganzen Reihe SAP-Freunde aus Brieg, die vor allem in der britischen und der Ostzone verteilt wurden, stehe ich in Verbindung. Der Fritz Lewy*, jetzt Lynn, wohnt auch in New York; wo mag Dr. Fritz Sternberg* sein? Leider konnte ich Oskar Krummschmidt noch nicht finden und Siegfried Pech dürfte wohl in Gefangenschaft sein. Seine letzten Grüße kamen 1944 von der Insel Rhodos. Daß Freund Oppler* in Wiesbaden ist, habe ich noch nicht gewußt. Konrad Reisner* hat mir auch geschrieben. Ja, wenn die Grenze der Ostzone endlich einmal fälth, dann dürfte an unserer Verständigung vieles besser werden. Erfreulich ist es immer wieder festzustellen, daß das alte Interesse überall lebt und an $\mathrm{Er}_{\mathbf{r}}$ fahrung reicher geworden ist.

Unsere einstige Parteijugendbewegung ist auch älter geworden und einer nach dem andern meldet sich bei mir. Sie waren alle gezwungene sehr schlechte Hitlersoldaten. Einer meiner tapfersten Brieger Freunde Karl Dierschke konnte 1936 der Gestapo entkommen, kämpfte 1937 in der 11 . int. Brigade in Spanien mit und fiel im September an der Aragonfront.

Gestern führten die Gewerkschaften in Bamberg und anderen Städten einen allgemeinen zweistündigen Proteststreik wegen der faschistischen Sprengstoffattentate durch, wobei ich hier in Betriebsversammlungen Ansprachen zu halten hattes. Lebhaft genug geht es bei uns zu. Verwunderlich ist das nicht, denn alles ist in einer Umformung begriffen und Widerstände sind dazu da, daß sie überwunden werden. Nach diesem alten Grundsatz haben wir weiter zu leben.

Lebt recht wohl und laßt Euch herzlich grüßen und für Eure vorbildliche kameradschaftliche Gesinnung in alter Treue danken

von Eurem Fritz Nagel und Familie

Fritz Nagel vermutlich an Konrad Reisner*

Bamberg, den 12.2. 1947

Memmelsdorferstr. 22

\section{Lieber Freund!}

Am 21. I. I 947 erhielt ich Deinen ersten Brief. An Dich zu erinnern vermag ich mich sehr gut. Du wirst doch O.K. und F. S.' gekannt haben und diese beiden haben Deinen Namen mir gegenüber manchmal genannt. Was war das für eine schöne, für uns alle lebhaft bewegte Zeit, als wir als Jungsozialisten und später als SAP-Genossen uns so manches Mal zum Meinungsaustausch in Breslau trafen. Menschen unserer Art konnten sich 1933 niemals so ohne weiteres einer kampflosen Resignation hingeben und den Nazis das Feld so ohne weiteres überlassen. Ich habe in Brieg und in zwei Orten des Landkreises die SAP illegal weitergehalten; dazu kam eine in der Illegalität noch sehr

\footnotetext{
' $\mathrm{Zu}$ den Protestaktionen in Bayern wegen der Sprengstoffanschläge vgl. Anm. 3 zum Brief Bernhard Molz' vom 5. 2. 1947, S. 120.

' Gemeint sind höchstwahrscheinlich Oskar Krummschmidt und Frizz Sternberg".
} 
starke Gruppe des soz.[ialistischen] Jugendverbandes, deren Existenz die Gestapo nie erfahren konnte auch als 1936 der ganze Bezirk aufgerollt wurde. Nur der Zufall konnte, wie mir selbst Gestapoleute sagten, ihnen gegenüber helfen unsere illegale Bewegung zu fassen. Die Zuverlässigkeit unserer Genossen war vorbildlich.

Dieser Idealismus wurde von der Gestapo deshalb als besonders gefährlich bezeichnet, weil er nicht stur geleitet war. Hätten wir uns dabei z. B. mit der KPD eingelassen, hätten wir keine drei Monate arbeiten können, weil diese Organisation damals von Spitzeln wimmelte. Daß uns auch unser Schicksal ereilte, lag, wie gesagt, nicht an uns. Bei einer lächerlichen Gepäckkontrolle nach geschmuggelter Butter im Postauto an einem schlesischen Grenzdorf fanden die Zollbeamten im Rucksack unseres Genossen Gremmel, der damit türmen wollte, an die 500 frisch aus Prag geholte verbotene Zeitungen und eine Anzahl Braunbücher'. Nun kannst Du Dir alles weitere ausmalen. Allerdings hat uns die nachfolgende Zeit schwer mitgenommen, vor allem auch Frau und Kinder, wenn ihr Ernährer auf Jahre im Zuchthaus festgehalten wird. Zur Nachkur holte man mich nach dem 20.7. 44 noch einmal ins $K Z$, aus dem ich durch die USA Truppen gerade noch im letzten Augenblick befreit wurde. Leider gingen diese Schicksalsschläge an mir nicht spurlos vorüber; sie haben mich etwas sehr arg mitgenommen. Trotzdem gebe ich mir Mühe meinen Platz als Funktionär der Arbeiterbewegung und Gewerkschaft auszufüllen, denn jeder von uns aus der alten Schule wird bitter gebraucht, angesichts der vielen Aufgaben, die infolge der Neubildung der politischen Fronten sich ständig stellen.

Es ist für mich eine große Freude wenn ich von Dir höre, daß Du und die übrigen Freunde uns drüben nicht vergessen habt und ich weiß auch, daß diese Hilfeleistung von Euch Opfer fordert, die bestimmt nicht klein sind. Für Deine Bemühungen um meine Person kann ich Dir leider nur auf diesem Wege danken, ebenso Mr. Peterson. Die Kleiderfrage, so peinlich sie ist, vor allem der Mangel an Unterwäsche, lieber Freund, spielt bei uns eine große Rolle. Die Textilien, die es mitunter auf einen Bezugsschein gibt, für Männer z. B. niemals Oberkleidung, sind von schlechter Qualität, daß von einer Behebung des Notstandes nicht eher gesprochen werden kann, bis bei uns wieder gute Rohstoffe an Wolle und nicht deutscher Wald verarbeitet werden. Bis dahin wird aber noch viel Wasser ins Meer fließen. Meine Mädels sind 22 und 23 Jahre alt. Ihnen wie meiner Frau fehlen vor allem Strümpfe, Unterwäsche und einiges Gummiband, das hier unmöglich zu bekommen ist. Mein ganzes Hab und Gut habe ich durch die Zwangsevakuierung meiner Familie aus Brieg nach Mecklenburg verloren. Was ich auf dem Leibe trug, wurde mir mit allen Wertsachen im $\mathrm{KZ}$ abgenommen. Bei meiner Entlassung erhielt ich einen Drillich-Turnanzug und ein Paar Filzstiefel mitten im Sommer. Deshalb, gebraucht wird jedes Kleidungsstück in diesem Lande der Trümmerhaufen.

Eine neue Möglichkeit ist Euch drüben auch jetzt gegeben, Sendungen mit Büchern und Schriften nach hier zu senden. Leider herrscht auch darin bei uns eine mächtige Knappheit. An volkswirtschaftlicher und sozialwissenschaftl. Literatur, gerade was wir

\footnotetext{
2 Herbert Gremmel (oder Grämmel) und Erna Krüger, die beide als Grenzkuriere arbeiteten, wurden im November 1935 festgenommen; ihre Verhaftung war der Ausgangspunkt für die Zerschlagung der Breslauer SAP 1936. - Das „Braunbuch über Reichstagsbrand und Hitlerterror" erschien am 1.8. 1933 in Paris als Gemeinschaftswerk deutscher Antifaschisten innerhalb und außerhalb Deutschlands Es setzte sich mit dem Reichstagsbrand auseinander und enthielt eine zusammenfassende Dokumentation über den Terror seit dem 30. I. I933. Innerhalb weniger Monate wurde das "Braunbuch" in I7 Fremdsprachen übersetzt.
} 
brauchen, wird nichts hergestellt, höchstens belletristische Zeitschriften oft undefinierbarer Art, die uns nicht interessieren. Die Geistesöde des Hitlerregimes hat auch auf diesem Gebiet ihre Wirkungen hinterlassen, dazu kam, daß die Gestapo auf unsere Bibliotheken, die wir uns früher mühselig aufbauten, ein scharfes Auge hielt. Die meine wurde von dieser Bande fast völlig weggeschleppt. So stehen wir also auch in dieser Beziehung nackt und bloß da.

Daß Kurt Oppler*, an den ich mich sehr gut erinnere, er war ja auch bei uns in Brieg, in Wiesbaden tätig ist, habe ich auch erst jetzt von Jola gehört und so werde ich versuchen mit ihm Vertindung zu bekommen. Wo mag unser Freund Kaliski hingekommen sein; er war so viel ich mich erinnere noch in Holland beim Einmarsch 1940 und kam von dort in ein KZ in Deutschland. Er mag wohl da zugrunde gegangen sein wie Karl Mache und der Ökonom Philipp des Gewerkschaftshauses, dessen Sohn 1933 von der SA erstochen wurde ${ }^{3}$. Der frühere Polizeipräsident Voigt und Präsident Wiersich vom ADGB sind 1944 in Berlin erhängt worden wegen Mitbeteiligung am 20. Julit. Da ich wehrunwürdig war, kam ich für die Wehrmacht nicht in Frage. Trotzdem bin ich durch eine Knochenverletzung am rechten Schambeinast während meiner Strafhaft 1936-39 heute zu über $70 \%$ körperbehindert worden und kann mich nur mühsam an zwei Stökken gehend fortbewegen. Das hat mir das Hitlerregime eingebracht. Doch deshalb lasse ich den Mut nicht sinken. Ich bin z. Zt. als Angestellter im Arbeitsamt tätig. Natürlich wäre ich lieber in unserem schönen Schlesien, als im schwarzen Lande der Bajuwaren, von dem ich mir früher auch andere Vorstellungen gemacht hatte. Die sprichwörtliche Intoleranz und Unduldsamkeit der hiesigen Bevölkerung uns Flüchtlingen aus dem Osten gegenüber bekommen wir bei jeder sich bietenden Gelegenheit zu spüren. Deshalb ersehnen wir nichts mehr als einmal wieder in unsere alte Heimat zurückzukehren. Über das Schicksal Deutschlands sind ja die Würfel noch nicht zum Stehen gebracht worden, sie rollen ja noch. DaB ein Nationalsozialismus noch einmal bei uns entstehen könnte, ist unmöglich; trotzdem versuchten so viele Profitmacher unter dem Mantel der Sicherheitsforderung ihre Vorteile zu wahren. Man kann auch von hier aus sehr gut durch den Schleier hindurchsehen, der über Tatsachen gebreitet wird. Dr. Schumacher führt in dieser Hinsicht eine offene Sprache im Interesse der Arbeiterklasse, die Chauvinisten von anderer Seite ihm als Nationalismus ankreiden. Doch wir verstehen schon schwarz von weiß zu unterscheiden. Wir Antifaschisten gehen auch auf das Gerede von der Kollektivschuld nicht ein, denn wir wissen am besten, was wir gegen den Nationalsozialismus angestellt haben. Unsere internationale Gesinnung ist

\footnotetext{
3 Der Jurastudent und Referendar Martin Kaliski, der der SAP in Breslau angehörte, emigrierte im Sommer 1935. Nach dem Einmarsch der deutschen Truppen in Holland wurde er verhaftet und kam später im KZ Mauthausen oder in Auschwitz ums Leben. - Karl Mache (1880-1944), ein Bäckergeselle, war in den zwanziger Jahren Funktionär der Breslauer SPD, Vorsitzender des SPD-Bezirks Breslau, mehrere Jahre Abgeordneter des Schlesischen Provinziallandtags und des Reichstags sowie bis 1933 2. Bürgermeister von Breslau. Im Herbst 1944 wurde er im KZ Dürrgoy ermordet. - Der Leiter des Breslauer Gewerkschafushauses Karl Philipp starb i945 unmittelbar nach seiner Befreiung aus dem KZ Dachau.

${ }_{4}^{4}$ Fritz Voigt (1882-1945): Bauarbeiter, I90s SPD, Gewerkschaftssekretär, 1918 Vorsitzender des Zentral-Soldatenrats für Schlesien, 1919 Mitglied der Nationalversammlung. 1919-1920 Polizeipräsident in Breslau, dann Leiter der schlesischen Bauhütten. Von März bis Dezember 1933 im KZ Lichtenburg. Wegen seiner Beteiligung am 20. Juli 1944 wurde Voigt am I. 3. 1945 in Berlin-Plötzensee durch den Strang hingerichtet Oswald Wiersich (1882-1945): vor 1914 SPD-Parteisekretär, Funktionär des DMV, in den zwanziger Jahren Bezirkssekretär des ADGB für Schlesien und Mitglied des preußischen Staatsrates. Wiersich wurde wie Voigt am 1. 3. I945 hingerichtet.
} 
trotz des Geistesterrors der Nazis lauter und rein geblieben und wir haben keinen größeren Wunsch als daß man uns dafür Verständnis entgegenbringt. Der Faschismus ist eine gewaltige Gefahr und Macht gewesen und niemand wußte es besser als wir, daß auch seine Stunde einmal schlagen mußte. Ich sehe noch heute im Geiste unseren Freund Ernst Eckstein* auf einer mächtigen Einheitsfrontkundgebung die wir in Brieg noch am 7. Februar 1933 abhielten, also nachdem die Nazis schon über eine Woche regierten, die prophetischen Worte sprechen, daß wenn von Hitler und seiner Bewegung kein Stäubchen mehr vorhanden sein wird, unsere Sache und unser Ziel noch viel fester und klarer vor der Menschheit stehen werden. Und so kommt es auch.

In diesem Sinne, lieber Freund, grüße ich Dich und alle anderen Kameraden auf das Herzlichste und wünsche Euch alles Gute.

Fritz Nagels.

Fritz Nagel an Joseph Lang

Bamberg, Ende Juni 1947

Memmelsdorferstr. 2a

Lieber Freund Jola!

Nun ist es hohe Zeit auf Deine Briefe vom 2. 3. und 2I. 4. zu antworten. Besonders danken aber muß ich Dir für die zwei Pakete, die meine Frau und Tochter kürzlich von Freund Katel' aus München holte. Eines war ausschließlich mit Lebensmitteln, das andere mit den verschiedensten Kleidungsstücken gefüllt. Das, was ich nicht unbedingt brauchte, gab ich an andere hilfsbedürftige Antifaschisten weiter. Jedenfalls war die Freude über Eure Mühewaltung bei uns sehr groß und die Lebensmittel sind in dieser kritischen Zeit, die unsere Kalorien pro Woche um fast die Hälfte kürzte, uns besonders willkommen. Das Brot wird fast ganz aus Maismehl hergestellt, Fleisch gibt es nur noch 100 und Butter keine $4 \circ \mathrm{gr}$. mehr auf die Woche. In einem Dorfe des Kreises rotteten sich am I. Pfingstfeiertag die Flüchtlinge zusammen und verlangten vom Bürgermeister Brot oder sie stürmen die Bauernhöfe und holen sich die vielen Festkuchen. Darauf ging der Bürgerıneister zu den Bauern und forderte sie zum Abliefern auf. In ihrer Angst erklärten sie sich natürlich sofort dazu bereit und die Flüchtlinge gingen befriedigt nach Hause.

Deine liebe Erna hat sich für mein Befinden sehr interessiert und ich kann ihr mitteilen, daß ich wieder kürzlich in der Univ. Klinik Erlangen zur Nachuntersuchung war und die Ärzte ein Stagnieren meiner Knochenaufbeulung am rechten Schambein, das allerdings im Ring zur Hälfte zerstört ist, feststellten. Ursache der Erkrankung war eine Verletzung des Knochens durch Schlag oder Stoß im Strafgefangenenlager Roßlau und eine an der Stelle sich entwickelnde Wucherung ist einmal wegoperiert worden, doch ein zweiter, den Schaden endgültig behebender Eingriff ist durch meine KZInhaftierung illusorisch geworden und hat mein Leiden so verschlimmert, daß ich stark lahm gelegt bin. Nach vier Monaten muß ich mich erneut in der Klinik vorstellen und man erhofft eine Verkalkung des Krankheitsherdes. Eine erneute Operation ist nicht

s Dieser Brief liegt nur in einer zeitgleich angefertigten Abschrift vor, die zu Langs gelangte.

' Der Sozialarbeiter russischer Herkunft Jacques Katel war nach 1945 als Vertreter des IRRC in Deutschland tätig. 
mehr möglich, auch neue Röntgenbestrahlungen sind vorläufig nicht erforderlich. Die fast ständig mehr oder weniger auftretenden Schmerzen bekämpfe ich durch schmerzlindernde Mittel, die in den Apotheken (was fehlt denn nicht alles) kaum zu kriegen sind. Um zu meinen häufigen Sitzungen und Besprechungen zu gelangen benütze ich möglichst Kraftwagen. In den nächsten Wochen ziehe ich endlich in eine eigene Wohnung ins Gewerkschaftshaus Kleberstr. 33a um, da habe ich die Büros in nächster Nähe, bisher wohnte ich als Untermieter möbliert. Da bei uns ein größeres Gewerkschaftssekretariat eingerichtet wird, hat man auch mir nahe gelegt mich als Sekretär zu bewerben. Doch befinde ich mich beim Arbeitsamt in angenehmer Stellung und habe dabei trotzdem die Möglichkeit mich in der Bewegung zu betätigen. Von der Gewerkschaft bin ich auch in den Verwaltungsrat des Verbandsvorstandes in München gewählt worden. Durch sie komme ich hier an Menschengruppen heran, an die hier in der schwärzesten Ecke Bayerns durch die Partei nicht heranzukommen ist. [...] Die Abhandlungen von P.[aul] F.[rölich] ${ }^{*}$ über die Sowjetunion und die Gewerkschaftsfrage habe ich auch erhalten. Seine Standpunkte teile ich durchaus und bin erstaunt darüber wie richtig z. B. P.F. die Bedeutung und die Wirkungsmöglichkeiten der heutigen Gewerkschaften bei uns beurteilt. Das Erfreuliche ist, daß die einstige Vielheit der Berufsverbände einer restlosen Vereinheitlichung der Gewerkschaften in der U.S. Zone in Industrieverbänden gewichen ist, zum Ärger aller der Kreise, die früher auf der Seite der christlichen Gewerkschaften standen und heute wieder mit Spaltungsabsichten liebäugeln. Richtungsmäßig sind hier die Gewerkschaften, die in Bayern an die 600000 Mitglieder umfassen, auf eine sozialistische Zielsetzung ausgerichtet. Es herrscht also das alte freigewerkschaftliche Element. Der Einfluß, den die Gewerkschaften in der Privatwirtschaft haben, ist größer als bei den Behörden. Ja, oft einflußreicher als vor 1933. Das ist zum guten Teil darauf zurückzuführen, daß der Betriebsrat Einfluß hat auf die Billigung der Beschäftigung Belasteter des Naziregimes und häufig Treuhänder an Stelle der alten Unternehmer stehen, aber auch wohl vor allem deshalb, weil das Unternehmertum seine organisatorische Kampffront noch nicht gebildet hat. Sie ist erst in einer langsamen Bildung begriffen. Aber auch weil die wirtschaftlichen Großunternehmungen des Kapitals aufgelöst sind und so die führenden Spitzen fehlen. Die rechtliche Stellung der Betriebsräte ist durch das Kontrollratsgesetz Nr. 22 (sogen. Betriebsrätegesetz) denkbar schwach. Das Gesetz besteht fast nur aus Kannbestimmungen. Trotzdem haben die Betriebsräte dort, wo zielbewußte Kollegen stehen, groBen Einfluß. Allerdings werden Erfolg versprechende Möglichkeiten sehr schnell durch die Besatzungsbehörden in der Westzone beseitigt und dem Wirtschaftsaufbau oft unvorstellbare Hemmungen entgegengesetzt durch die unsinnigsten Maßnahmen. So liegt jetzt z. B. unsere Bauwirtschaft in der schönsten Zeit des Jahres völlig darnieder, weil bis zum 30. Juni alle hergestellten und herzustellenden Baustoffe von der Mil. Reg. für ihre $Z$ wecke beschlagnahmt sind. So sind auch große Lager von Textilstoffen und anderen Waren seit I945 nach wie vor beschlagnahmt, während die Bevölkerung ihre halbzerstörten Häuser nicht aufbauen kann und,Bekleidung kaum zu haben ist. Die Folge ist eine Schieberei mit allen möglichen $W$ aren und Baustoffen die als Kompensationsgeschäfte sogar von den Behörden zugelassen sind. Z. B. erhalten die zwei Schuhfabriken in Bamberg $20 \%$ ihrer Produktion, um aus der anderen Zone Garne, Nägel und sonstige Zutaten zur Schuhherstellung zu tauschen. Aus dem Ruhrgebiet kommen Händler mit den so sehr benötigten Eisenwaren nach hier, um bei den Bauern Lebensmittel einzutauschen. Wir leben wie in der Naturalwirtschaft. Bedauernswerte Geschöpfe sind demgegenüber alle Lohn- und Gehaltsempfänger, die ihre wenigen Geld- 
scheine und sonst nichts in den Händen haben. Zur Kontrolle und Überwachung dieser Zustände versuchen sich auch die Gewerkschaften einzuschalten. Infolge des Lohnstopps sind sie nicht in der Lage Lohnverhandlungen zu führen, dafür kämpfen sie um Schwer- oder Teilschwerarbeiterzulagen bei den Ernährungsämtern. Da und dor flakkern wilde Streiks auf und bleiben nicht ohne Wirkung. Allmählich werden auch die Arbeitsgerichte, die Spruchkammern bei den Arbeits- und Versicherungsämtern wieder eingeführt und ein weiteres, immer mehr an Bedeutung für die Gewerkschaften gewinnendes Arbeitsgebiet bildet das Genossenschaftswesen. Leider sind die hier 1933 von der Arbeitsfront geraubten gewaltigen Kapitalsanlagen von der Mil. Reg. den Gewerkschaften noch nicht zurückgegeben, wie überhaupt die Frage der Wiedergutmachung auch für die Opfer des Faschismus ganz ungenügend ist.

Im Parteileben brachte die vor kurzem in Landshut stattgefundene Landeskonferenz der SPD die Ausbootung Hoegners aus dem Parteivorstand. Auf der örtlichen Parteiausschußsitzung habe ich es durchgesetzt, daß die hiesigen drei Delegierten gegen die Fortsetzung der Koalition stimmten ${ }^{2}$. Der Wortführer der Opposition war unser Freund Mar* aus München. Bedeutungsvoller kann unser Wirken erst werden, wenn die Zonengrenzen fallen und die Besatzungsmächte nicht die Durchführung von Beschlüssen verhindern werden, die im Zuge der Umformung der Wirtschaft im sozialistischen Sinne liegen. Was nützt uns die Beherrschung des bizonalen Wirtschaftsamtes in Minden ${ }^{3}$, praktisch dürfen wir nichts unternehmen was die kapitalistische Wirtschaft in Deutschland einengt. Die Behörden arbeiten oft gegeneinander, eine neue Verfügung hebt die andere auf und Gesetze werden erlassen und die Ausführungsbestimmungen fehlen. In den Ausschüssen weiß keiner mehr was los ist und alles stiert auf den sich mehr und mehr verdunkelnden Horizont im Osten, der eine geradezu lähmende Wirkung zeitigt. Die meisten politischen Diskussionen kreisen um die Frage einer Auseinandersetzung zwischen Ost und West. Die Gewährung der Anleihen an Griechenland und die Türkei, das Verhalten der Russen Österreich gegenüber, das einer Sabotage des Friedens gleichkommt, die Regierungsänderung in Ungarn sind Fragen, die damit in Zusammenhang gebracht werden ${ }^{4}$. Trotzdem glaube ich nicht an ei-

${ }^{2}$ Am 9./10.5. 1947 fand in Landshut die 4. Landeskonferenz der bayerischen SPD statt, auf der Waldemar von Knoeringen als Nachfolger des zurückgetretenen Wilhelm Hoegner zum SPD-Landesvorsitzenden gewählt wurde. Es gab scharfe Kritik an der bisherigen Politik des Landesvorstandes und an der Regierungskoalition zwischen CSU und SPD unter Ministerpräsident Hans Ehard (CSU). Im September 1947 beschloß der SPD-Landesausschuß den Austritt der SPD aus der Regierung Ehard.

3 Seit Februar 1947 stellte die SPD mit Viktor Agartz den Direktor des bizonalen Verwaltungsamtes für Wirtschaft; Agartz war bereits 1945/46 Leiter des Zentralamtes für Wirtschaft in der britischen Zone gewesen.

4 Am 12.3. 1947 verkündete der amerikanische Präsident Truman die nach ihm benannte TrumanDoktrin, in der er allen nichtkommunistischen Ländern die Unterstützung der USA bei ihrer Auseinandersetzung mit dem Kommunismus anbot. Unmittelbare Folgen dieser Doktrin waren Kreditzusagen und Waffenlieferungen an Griechenland und an die Türkei. - In Österreich versuchte die Sowjetunion, in den Verhandlungen zum Abschluß eines Friedensvertrages ihre Forderung nach Übernahme der deutschen Guthaben und des deutschen Eigentums in Österreich als Reparationen durchzusetzen. - In Ungarn führte die Aufdeckung einer angeblichen Verschwörung gegen die demokratische Ordnung, in die neben Militärs vor allem führende Mitglieder der aus den Wahlen im November 1945 als stärkste Partei hervorgegangenen Kleinlandwirtepartei des Ministerpräsidenten Ferencz Nagy verwickelt waren, Ende Mai 1947 zum Rücktritt des Ministerpräsidenten und zur Umbildung der Regierung. Dadurch wurden der Einfluß der KP erheblich verstärkt und die Kleinlandwirtepartei als unabhängige Partei langsam ausgeschaltet; aus den Neuwahlen vom August 1947 ging die K.P als stärkste Partei hervor. 
nen Krieg. Der Russe führt sich eben so plump fordernd auf, weil er weiß, daß die anderen Mächte keinen Krieg deshalb beginnen werden. Genauso zeigt er sich im Sicherheitsrat, im Ausschuß für Atomkontrolle und überall dort, wo er mit seinem Vetorecht alles durcheinander bringen kann. Aber im Hinblick auf unsere Ernährungslage, die eine schnelle Bereinigung der außenpolitischen Situation erfordert, sind das alles Spielereien, die allen Beteiligten nur teuer zu stehen kommen, denn sie kosten viel Zeit, die nutzbringender verwendet werden könnte.

Eine zunehmend politische Bedeutung gewinnt bei uns die Vereinigung der Verfolgten des Naziregimes, die als eine übernationale Organisation sogar im Begriff ist von den Alliierten in der UN anerkannt zu werden. Diese Vereinigung umfaßt alle politischrassisch $u$. religiös Verfolgten. Ähnliche Vereinigungen sind in fast allen Ländern die Hitler schädigte entstanden, und schließen sich international vor allem zum Zwecke der Wiedergutmachung erlittener Schäden zusammen. Die Zahl der in Bayern lebenden politisch Verfolgten einschl. Flüchtlinge beträgt 18000 , dazu 17000 rassisch Verfolgte. Von z. B. 2000 Juden die früher in Bamberg lebten, existieren heute noch ganze 5. Das Jahr 1936 war das, in dem die meisten politischen Verhaftungen erfolgten und zwar 38-40000. Gar manche illegale Organisation, die damals häufig entstanden, hielt sich nur kurze Zeit und die Gestapo wand allerlei Tricks an um Genossen zu überführen. Im Ruhrgebiet gab es einige K.P. Gruppen, die bis 2500 Mann stark wieder in kleinere Gruppen aufgespalten vor die Strafsenate des Oberlandesgerichts Hamm i. W. zur Verurteilung kamen. Dieses Gericht war wegen seinen schweren Verurteilungen berüchtigt. Das Strafmaß steigerte sich von Jahr zu Jahr. Wofür ich z. B. 1936 drei Jahre Zuchthaus erhielt, gab es 1944-45 die Todesstrafe. Was hätten die Nazis wohl dazu gesagt, wenn sie je gewußt hätten, daß ich als junger Funktionär im September 1923 , als ich von Bayern zurückkam wo ich die ersten Anfänge der Hitlerbewegung kennen lernte, in aller Stille im Auftrage der Gewerkschaften in Brieg zwei Hundertschaften proletarischen Selbstschutzes organisierte, der mit Schußwaffen und Handgranaten, aus alten Weltkriegsbeständen zusammengeholt, gut bewaffnet war und am 8. u. 9. I I. 1923 in höchster Alarmbereitschaft lag um im Falle eines Putsches dieser Banditen bei uns sofort zuzuschlagen, ohne erst eine Generalstreikparole abzuwarten. Diese organisierte Selbsthilfe war leider schon damals eine streng verbotene Sache und ist nach $\mathrm{Zu}$ sammenbruch des Hitlerputsches in München wieder von uns demobilisiert worden um nicht unnötig, sagen wir einmal gute Demokraten, schon damals der Klassenjustiz auszuliefern, nachdem jeder von den 200 Mann seine Sache gemacht und dicht gehalten hatte. Ich freue mich noch heute wenn ich an diesen gelungenen Streich denke.

Während meiner Untersuchungshaft in der Graupe [Breslau] wurde eine Gruppe von etwa 80 Mann Schwarze Front' eingeliefert, dann Gruppen von Mönchen, Geistlichen wegen $\ 175$, Devisenschiebung u. ä., da war von der KPO die Gruppe Rektor Felsen, Breslau. Im Zuchthaus lagen wir in Brandenburg a. H. in jeder Einmannzelle 3 Mann. Dann wurden besondere Strafgefangenenlager eingerichtet, außer den K.Z.Lagern, denn man wußte nicht mehr wohin mit den vielen Gefangenen, dabei waren noch keine Ausländer darunter. Zahlreiche Gruppen von Gefangenen wurden, schrecklich untergebracht, beim Bau des Westwalls eingesetzt. Die meisten Verhaftungen erfolgten in den Jahren 1935-37. Dann war der innere Widerstand illegaler organisierter Gruppen

\footnotetext{
' Anhänger von Otto Strasser, der zusammen mit seinem 1934 ermordeten Bruder Gregor ein führender Verreter des linken Flügels der NSDAP war und nach seinem Austritt aus der NSDAP die „Kampfgemeinschaft Revolutionärer Nationalsozialisten“ gründete, aus der später die „Schwarze Front ${ }^{a}$ hervorging
} 
gebrochen. Er wurde nur in kleineren Kreisen fortgesetzt, die aber von der Gestapo gut beobachtet wurden. Mit dem Genossen Herbert Tulatz $z^{*}$ aus Breslau traf ich nach unserer Entlassung $194^{\circ}$ aus der Haft einigemal wieder zusammen ohne daß irgend jemand davon wußte, trotzdem wurde er bald erneut zur Gestapo zitiert und schwer vermahnt. Durch meine spätere Überführung ins K.Z.Gr. Rosen waren wir erneut auseinandergerissen. Heute finden wir uns, soweit wir noch leben, wieder zusammen. Schade daß die Grenze der Ostzone uns so scharf trennt, denn dort sitzen die meisten Bekannten und es sähe um uns manches anders aus. [...]

Ich hätte Dir ja noch vieles vieles mitzuteilen, aber ein Brief läßt dies nur in beschränktem Umfang zu.

Vielen Dank auch noch für die Zigaretten, die ich mir bei Freund Opel* in den nächsten Tagen holen werde. - [handschriftlich hinzugefügt:] Schon geschehen.

Nochmals herzlichen Dank für Deine viele Mühewaltung und die Fürsorge Deiner Frau.

Es grüßt Dich recht herzlich Dein Freund

Fritz Nagel und Familie

Fritz Nagel an Joseph Lang

Bamberg, den 19. 10. 1947

Kleberstr. $33 a$

Lieber Freund Jola!

Deine Briefe und Rundschreiben vom August und September habe ich erhalten. Daraus kann ich feststellen, welche umfangreiche Tätigkeit ihr drüben und vor allem Du mit dem Hilfswerk aus dem Solidaritätsfonds für uns hier geleistet habt. Mir ist gleichwertiges von anderen linkspolitischen Gruppen nicht bekannt. [...] Von mir aus muß ich sagen, daß Eure Hilfe vor allem auch in der Bekleidung für mich und meine Familie in vieler Hinsicht von entscheidendem Einfluß war. Was ich nicht selbst unbedingt brauchte habe ich davon unter Angabe der Herkunft an andere hilfsbedürftige Genossen weitergebeben. Da ich im Gewerkschaftshaus nun auch wieder eine eigene Wohnung beziehen konnte, waren die Textilien für mich besonders wertvoll. Auf welche Weise ich Euch dafür mit einer Gegenleistung dienen könnte weiß ich leider noch nicht. Vorläufig kann ich meinen großen Dank Euch nur immer wieder in dürren Worten aussprechen. Ich muß dabei immer an das Verhältnis Marx-Engels denken, deren über alles erhabene Freundschaft und Hilfsbereitschaft wie ein leuchtender Stern auf Euer Hilfswerk strahlt, das eine sozialistische Tat für sich darstellt. Das beweist Dein Bericht über Eure Aktion. Lieber Freund! Du wunderst Dich daß ich nicht schreibe! Einen Brief, glaube ich, mußt Du von mir in den letzten Wochen bekommen haben. Manchmal bin ich ganz verzweifelt, daß ich meine Post nicht regelmäßig beantworten kann. Wäre ich nicht so leidend, würde mir dies viel leichter fallen, doch die Mitarbeit an den Organisationen füllt die meisten meiner dienstfreien Stunden. Vergangene Woche z.B. Montag abend mußte ich einen Zeitungsartikel für den Ortsausschuß der Gewerkschaften gegen einen hiesigen Bürgermeister der CSU wegen der Kartoffelversorgung der arbeitenden Bevölkerung schreiben. Dienstag abend jede 2. Woche bei der Gewerkschaftsjugend im Rahmen eines laufenden Kursus über die Geschichte der Gewerkschaften sprechen. Mittwoch abend jede Woche in der sozialistischen Arbeitsge- 
meinschaft, in der ich SPD und KPD-Funktionäre zusammenfaßt habe (1931-32 wollte es nicht gehen, jetzt geht es) über den "Dialektischen Materialismus“. Donnerstag mußte ich im Bett bleiben wegen starker Beinschwellung. Freitag abend sprach ich in der Versammlung meines Parteidistrikts über: die weltpolitische Lage. Sonnabend endlich, Vorstandssitzung meiner Gewerkschaft. Das ist alles völlig „ehrenamtliche“ Arbeit und so geht es Woche um Woche. Angesichts der bestehenden Zustände kann ich mich nicht abseits stellen. Dies ginge gegen mein Gewissen und meine Überzeugung und $\mathrm{Ihr}$ würdet es drüben auch nicht verstehen können. Unsere politische Situation wird angesichts der bevorstehenden Demontagen immer verrückter. Die westlichen Demokraten wundern sich dauernd, warum bei uns die Demokratie nicht richtig Fuß fassen will. Wir wundern uns über diese Naivität, die geradezu kindlich anmutet. Wenn man uns Lebensmittel schickt, so ist das schön und gut, wenn man uns aber die Möglichkeiten nimmt diese zu bezahlen, das verstehe wer will. Ja wenn es sich wenigstens ausschließlich um Rüstungsbetriebe handelte, aber die allermeisten der Werke haben sich längst auf Friedenswirtschaft umgestellt und vor allem die Kraftwerke brauchen wir so notwendig. Das nennt sich „Aufbau“ der Wirtschaft wenn zwei Millionen Arbeitern die Produktionsstätten genommen werden. Die Demontage überschattet hier gegenwärtig alles und wirkt in Verbindung mit der Kohlen- und Kartoffelnot einfach niederschmetternd auf die Bevölkerung. Kein Wunder wenn dadurch nazistische Strömungen florieren und amtliche Stellen sich über die Bekämpfung antisemitischer Machenschaften unterhalten müssen. Auch der kleinste Mann spürt daß es hier nicht mehr um Zerstörung eines Kriegspotentials geht, das ist bereits geschehen, es ist nackte Ausschaltung der Konkurrenz vom Weltmarkt. Zuckerbrot und Peitsche zugleich angewandt, erzieht in der Demokratie ebenso wie in der Diktatur zur Heuchelei, das müßten auch Demokraten endlich lernen, die immer schnell bei der Hand sind, einem im Siegestaumel schwellenden Diktator nachzuweisen, daß er alle Vernunftgründe in den Wind schlägt.

Nun lieber Freund wünsche ich Dir und allen andern alles Gute, und vielen Dank auch für das gestern erhaltene Care-Paket.

Sei nun aufs herzlichste gegrüßt von Deinem

Fritz Nagel u. Familie

Fritz Nagel an Joseph Lang

Bamberg, den 20.3.1948

Kleberstr. $33 a$

Mein lieber Freund Jola!

Endlich komme ich dazu Dir Deinen lieben Brief vom 22. 2. zu beantworten. Viel Erfreuliches über mich kann ich Dir allerdings nicht mitteilen, denn seit Anfang Dezember muß ich im Bett liegen. Der Zustand der Krankheit erfordert, daß der Körper völlig stillgelegt wird, wenn überhaupt noch etwas gerettet werden soll, denn das rechte Schambein ist zerstört und der Beckenknochen mit dem Hüftgelenk muß erhalten bleiben. Ich habe mir eben bei meiner Tätigkeit im öffentlichen Leben zu wenig Ruhe gegönnt. Daß ich nun so untätig das Bett hüten muß, ist mir die reinste Qual, denn drauBen verlangt die Bewegung förmlich nach mir, ohne dabei zu übertreiben. Ich sollte z.B. unter allen Umständen jetzt auch für die Wahl zum Stadtrat kandidieren. Man 
rennt mir von der Partei aus fast die Bude ein. Aber was nicht geht, das geht eben nicht! Für Deine Sorge mich von meinen quälenden Schmerzen etwas zu befreien, danke ich Dir von Herzen. Die Medizin habe ich von Freund Opel* erhalten, nur schade, daß sie schon alle ist. Mein Arzt tut ja für mich auch was er kann und daß Ihr ihn durch eine Spende ehrt, darüber ist er hoch erfreut gewesen.

In dieser Woche war ich auch wieder zur Röntgenaufnahme in Erlangen, aber wie gesagt, ich stehe mit meinem Stand der Krankheit auf des Messers Schneide. Nun hat sich ja auch bei meinen übrigen Freunden in Deutschland mein Zustand herumgesprochen und alle nehmen großen Anteil an meinem Schicksal. Einer hat wohl die Sache schon ganz tragisch aufgefaßt und er ist der Meinung daß ich schon in die Unterwelt abgefahren wäre und so schrieb er meiner Frau einen schönen Beileidsbrief. $\mathrm{Na}$, man sagt immer wenn man tot gesagt wird bei Lebenszeiten, wird man noch lange leben.

Über die Verwendung Deiner Penicillinspende kann ich Dir nur mitteilen, daß sie im Krankenhaus doch nicht angewandt werden konnte, da der Fall anders lag als erst vermutet wurde und so befindet sie sich noch in den treuen Händen meines Arztes.

Wenn ich Dir nun einiges über die politischen Verhältnisse noch mitteilen darf, so folgendes: Das Erstarken der Gewerkschaften und ihre Einflußnahme auf das politische Geschehen wie es durch den Proteststreik am 2 I. Januar wegen der beabsichtigten Fettkürzung zum Ausdruck kam', ist der CSU furchtbar in die Glieder geschlagen. Durch dieses entschlossene Vorgehen der Gewerkschaften ist vor aller Welt die Unfähigkeit der derzeitigen Bayer. Regierung in der Ernährungsfrage klipp und klar festgestellt worden. Da ja die Bauernschaft zum größten Teil zur CSU steht, ist es erklärlich, daß die Regierung bei ihren Plänen in Punkto Einhaltung des Ablieferungssolls dauernd beide Augen zugedrückt hat. Die Folge ist, daß die Landbevölkerung $4 \times$ so viel zu essen hat als die Stadtbevölkerung. Dazu kommt, daß die SPD in voller Öffentlichkeit genaue Angaben machen konnte über die Art der Eierverteilung. Während die Bevölkerung fast keine zu sehen bekommt, werden die kirchlichen Einrichtungen wie Klöster, Internate, Pensionistenheime und die Küchen der kirchlichen Würdenträger mit großen Mengen beliefert. Das ist natürlich ein Skandal, der in der Öffentlichkeit großes Aufsehen hervorgerufen hat.

Ich hoffe, daß die hier sich neu bildende Bayernpartei der CSU bei den nächsten Wah. len schweren Schaden zufügen wird. Wie sich der in Frankfurt neu gebildete bizonale Wirtschaftsrat auf die Belebung unseres Wirtschaftslebens in der Westzone bewähren wird, darauf kann man gespannt sein. Ja, wenn er frei von der amerik. Wirtschaftskontrolle wäre, denn diese hat sich bisher nicht so fördernd ausgewirkt wie wir es uns wünschten. Drüben in der Ostzone redet man nur noch von der Sklaverei wie sie jetzt durch den Marshallplan über die westeuropäischen Mächte kommen wird. Dabei sieht es in der Ostzone mehr als traurig aus. Die SED kann machen was sie will, ihr Volkskongreßgedanke findet hier keinerlei Gegenliebe, da auch der einfachste Mensch fühlt, daß dieses Gebilde uns dem Frieden auch nicht einen Zoll näher bringt ${ }^{2}$. Ich habe das

\footnotetext{
' Gemeint ist der vom Bayerischen Gewerkschaftsbund wegen der schlechten Ernährungslage ausgerufene Generalstreik, der am 23. I. 1948 in ganz Bayern durchgeführt wurde und an dem sich mehr als eine Million Arbeiter und Angestellte beteiligten. Vgl. dazu auch Anm. 2 zum Brief Karl und Emma Grönsfelders vom 24. 2. 1948, S. 210.

${ }^{2}$ Am 26. i r. r 947 lud die SED alle Parteien aus allen Besatzungszonen zu einem "Deutschen Volkskongreß für Einheit und gerechten Frieden" für den 6./7. 1 2. 1947 nach Berlin ein. Aus den Westzonen sagte als einzige Partei die KPD zu, allerdings nahmen an dem Kongreß auch Einzelpersonen
} 
Gefühl als ob die Teilung Deutschlands, so wie sie jetzt besteht, nicht mehr rückgängig gemacht wird, denn wie es in einem Deutschland zugehen wird in dem private Großbetriebe, die mit dem internationalen Kapital verflochten sind, private Mittelbetriebe, Produktionsgenossenschaften, landeseigene Betriebe und sowjeteigene Betriebe nebeneinander wirtschaften sollen, dazu die ungeheuer großen Unterschiede in der reformierten Landwirtschaft, ist mir völlig unklar. Man wird eben bei den Verhandlungen unter diesen Umständen nie zu einem Ergebnis kommen und die Ostzone wird die Republik Nr. 17 der UdSSR. Mit Gewalt läßt sich eben das alte Deutschland nicht mehr herstellen, aber unter dem jetzigen Zustand kann es die Arbeiterklasse auch nicht länger aushalten. Unseren alten Lebensstandard wieder zu erhalten, wird noch langer zäher Kämpfe bedürfen.

Nun lieber Freund, auch $\mathrm{Du}$ und $\mathrm{Ihr}$ anderen über dem großen Wasser werdet Eure Sorgen haben. Ich wünsche Euch alles Gute und Erfolge in Eurem Lebenskampf.

Die herzlichsten Grüße sendet Dir und allen Freunden

Euer Fritz Nagel u. Familie?

\section{Otto Wemer Mager* an den Solidaritäts-Fonds}

Bamberg, den 5. 2. 1948 Schützenstr. 7

\section{Liebe Genossen!}

Über Euer Lebensmittelpaket habe ich mich so von Herzen gefreut, denn es sind ja nun schon so viele Jahre her, wo wir gemeinsam kämpften und wir, die wir hier geblieben sind, eine schwere Zeit durchmachen mußten. Man verhaftete uns, nahm uns in Schutzhaft und sperrte uns dann schließlich ins Gefängnis. Nun ist diese dunkle Zeit vorbei, und trotzdem stehen wir vor schier unüberwindlichen Schwierigkeiten. Aber trotzdem sind wir wieder die alten und stehen nun wieder am alten Fleck wie vor 1933. Wir sind unermüdlich tätig, die letzten Reste des Nazismus zu beseitigen, und sind bemüht die Arbeiterschaft in eine geschlossene Front zu bringen. Aber man hat vorläufig

teil, die anderen Parteien angehörten. Der „1. Deutsche Volkskongreß“ verabschiedete ein Manifest an die in London tagende Konferenz der Außenminister der vier Allierten. Nach den Vorstellungen von SED und KPD sollte die Volkskongreßbewegung eine gesamtdeutsche Massenbewegung werden; die KPD plante für Anfang 1948 Volkskongresse in den einzelnen Ländern der Westzonen, die aber 1948 von den westlichen Besatzungsmächten verboten wurden. Die KPD erreichte aber ohnehin ihr Ziel nicht, über die aktuellen Themen deutsche Einheit und Friedensvertrag Mitglieder der SPD und der nicht-sozialistischen Parteien für die Volkskongreßbewegung zu gewinnen und auf diese Weise ihren Einfluß in den Westzonen wieder zu stärken.

3 Am 10. 5. 1948 schrieb Helene Nagel an Joseph und Erna Lang:

Liebe Familie Lang, liebe Gesinnungsfreunde!

Schweren Herzens muß ich Euch mitteilen, daß mein lieber Mann, Euer aufrechter Freund nach einem schmerzensreichen Leben am 6. Mai verschieden ist [ . . . Nun ist er erlöst und so haben wir seinen Leib am 8. Mai in die kühle Erde gebettet. Es war eine wirklich ehrenvolle Beisetzung, ein kleines Ereignis für Bamberg, denn wir sind konfessionslos und so war kein Pfarrer anwesend, sondern ein lieber Freund Emil Deutsch* hielt die Trauerrede, oder besser gesagt, widmete ihm einen herzlichen Nachruf. Viele Organisationen waren vertreten und taten mit herzlichen Worten und reichen Kranzniederlegungen ihr Beileid kund. [...] Euch aber ihr Lieben alle, die Ihr ihm und uns so unendlich viel Gutes getan, ruft Euer toter Freund einen herzlichen Abschiedsgruß und ein herzliches Lebewohl zu. $[\ldots]$ 
noch nicht viel gelernt, denn das gegenseitige Bekämpfen ist wieder im vollen Gange. Unsere erste Aufgabe muß es deshalb sein, eine einheitliche und geschlossene Gewerkschafusbewegung zu schaffen, um gegen die auflebende Reaktion gewappnet zu sein. Wir haben da schon beachtliche Erfolge erzielt. Wir wollen nun diejenigen sein, die für den Frieden kämpfen, damit nicht noch einmal das Unglück über uns hereinbricht. Denn die Nachwirkungen des wahnsinnigen Krieges sind fürwahr schrecklich. Vor allem der brave Arbeiter, der alle seine Kräfte zum Wiederaufbau einsetzt, muß arg darben, während die Schieber, Schwarzhändler und Nichtstuer ein herrliches Leben führen und die deutschen Stellen nicht genügend durchgreifen. Deshalb hatten wir kürzlich hier in Bayern 24 Stunden Arbeitsruhe, welche in aller Ruhe und Disziplin durchgeführt wurde'. Unsere Aufgabe ist schwer, und doch müssen wir es schaffen. Deshalb war ich tief beeindruckt, als ich das Paket von Euch erhielt. Denn es war mehr als ein Paket. Es war ein Zeichen, daß Ihr uns in unserer schweren Zeit doch nicht vergessen habt. Allen Genossen nochmals meinen herzlichsten Dank!

Mit herzlichen Grüßen

Werner Mager

\section{Otto Werner Mager an den Solidaritäts-Fonds}

Bamberg, den 1 2.7. 1948 Schützenstr. 7

Liebe Genossen!

Vor einigen Tagen erhielt ich Eure so netten Zeilen. Ich freue mich jedesmal sehr, wenn ich von Euch wieder eine Antwort erhalte. Habt recht herzlichen Dank dafür.

Vom Tod Fritz Nagels" habt Ihr ja Nachricht erhalten. Er hatte eine wunderbare letzte Fahrt. Die Beteiligung der gesamten Bamberger Arbeiterschaft war sehr groß und die Grabreden wollten gar kein Ende nehmen. Man hat gesehen, daß Fritz eben überall sehr beliebt war. Ich komme sehr oft mit seiner Frau und seiner Tochter zusammen.

$[\ldots]$

Bei uns hier hat sich in den letzten Wochen auch so allerhand ereignet. Nun ist endlich die langersehnte Währungsreform in Gang gekommen. Nur hatten wir gehofft, daß die kleinen Sparer und braven Arbeiter etwas berücksichtigt würden, aber leider sind wir enttäuscht worden. Vor allem sind wir sehr erstaunt, daß es auf einmal so viele Waren gibt, welche wir seit Kriegsende nie zu Gesicht bekommen haben. Es ist eben alles gehortet worden. Die Preisüberwachung ist auch teilweise aufgehoben worden und deshalb das rapide Steigen derselben. Mag manches für uns Schaffende auch schwer sein, besonders die Überwindung der momentanen Übergangszeit, so sind wir doch froh, daß unsere Arbeit jetzt wieder an Wert gewinnt, denn man kann sich jetzt auch wenigstens etwas kaufen. Die Ernährungslage hat sich in der letzten Zeit auch wesentlich gebessert, vor allem da es jetzt sehr viel Gemüse gibt. Auch haben wir gegenüber vorigem Jahr ausgiebig Regen. Wir denken, daß es doch nun besser werden wird. Gerade in der letzten Zeit war die Lage der Arbeiterschaft fast hoffnungslos und es kostete unsere

\footnotetext{
' Gemeint ist der 24stündige Generalstreik in Bayern am 23. 1. 1948; vgl. dazu Anm. 1 zum Brief Fritz Nagels vom 20. 3. 1948, S. 232 ; sowie Anm. 2 zum Brief Karl und Emma Grönsfelders vom 24. 2. 1948, S. 210.
} 
ganze Mühe, daß sie vernünftig blieb. Der Radikalismus nahm oft beängstigende Formen an. Auch nahmen die separatistischen Bewegungen innerhalb Bayerns stark zu. Nun ist durch die Berliner Krise wieder eine neue schwierige Lage entstanden. Unser Wunsch und Ziel ist und bleibt es danach zu streben, daß recht bald ein einheitliches Deutschland wieder erstehen möchte, denn sonst wird es bei uns keine Ruhe geben. Die Russen haben eben andere Ziele und da gehen sie keinen Schritt davon zurück. Wir wollen nur hoffen, daß uns endlich nach so vielen Jahren des Jammers und der Not endlich einmal Friede wird. Wir wollen als schaffende Menschen unsere ganze Kraft dafür einsetzen, verlangen aber, daß wir mitarbeiten dürfen, was durch unsere $\mathrm{Ge}-$ werkschaftsbewegung erfolgen müßte. Nur glaube ich, daß eines Tages doch die einheitliche Gewerkschaftsbewegung in die Briche gehen wird und wir wieder zu dem alten System zurückkehren, anstatt die Lehre aus Vergangenem zu ziehen. Der Arbeiter hier in Bayern ist eben ein anderer Schlag als in der fruheren Heimat. Wollen wir hoffen, daß doch einmal eine bessere Zeit auch für uns wieder einmal kommen wird. Wir wollen unsere ganze Kraft dafür einsetzen.

Liebe Genossen! Habt Dank, daß Ihr meinen Namen mit nach der Schweiz gegeben habt.

Ihr schreibt mir auch, wenn ich einmal etwas an Bekleidung benötigen sollte, dann möchte ich es Euch mitteilen. Mir liegt das Betteln nicht, und wenn ich mit einer Bitte komme, dann wird es sicher die einzige sein. $\mathrm{Da}$ ich auch alles verloren habe, fehlt es mir an einer Hose und Jakett. Sollte mein Wunsch Euch nicht zuviel sein, so danke ich Euch schon herzlichst im Voraus. Meine Größe beträgt 1,78 mtr. Die Anzugsgröße kann ich Euch leider nicht angeben, da es schon fast nicht mehr wahr ist, seitdem ich mir den letzten Anzug kaufen konnte. Meine Figur ist schlank wie bei allen Arbeitern. Habt für Euren Brief nochmals recht herzlichen Dank und die besten Grüße und Wunsche sendet Euch

Euer Werner Mager

\section{Otto Wemer Mager an den Solidaritäts-Fonds}

Bamberg, den I5.8. 1949 Schützenstr. 7

\section{Liebe Freunde!}

Nun wird es aber höchste Zeit, daß ich Euch wieder einige Zeilen schreibe. Euer Paket haben wir mit großer Freude erhalten. Unterdes ist uns eine Tochter, Irene Brigitte, geboren worden. Obzwar ich lieber einen Jungen gehabt hätte, bin ich trotzdem sehr froh, denn es ist alles gesund und munter. Das Töchterchen macht jeden Tag neue Fortschritte und es bereitet einem täglich immer mehr Freude. Ich glaube, daß Ihr eine gute Nase gehabt habt, daß es eine Tochter geworden ist, denn Ihr schicktet uns ein paar nette Kleidchen. Wir haben diese bereits schon angezogen, und da sieht sie allerliebst aus. Ihr habt uns damit eine sehr große Freude bereitet. Auch meine Nichte, das Flüchtlingsmädel, hat sich über die Kleider sehr gefreut und wird Euch ein paar Zeilen mit beilegen. Für sie ist es ja doppelt schwer, da sich ihre Eltern noch im polnisch besetzten Teil von Oberschlesien befinden. Wir haben uns ihrer angenommen, denn da hat sie wenigstens einen Halt und ein Zuhaus. Ich selbst bin ja noch nie ein Egoist gewesen und habe immer geholfen soweit es mir selbst möglich war. Nun hat sich auch 
unsere Wohnraumlage etwas gebessert, denn wir haben noch ein Zimmer dazu bekommen und leben für uns in dem Hause abgeschlossen von den anderen Mietern. Es gibt eben heute noch sehr viele unvernünftige Menschen, mit denen man am liebsten nicht in Berührung kommen möchte. Die Not und das Elend waren eben nach diesem Krieg zu groß. Unser Los hat sich seit der Währungsreform nun doch in vielem gebessert. Jetzt gibt es wenigstens wieder genügend zu essen, und auch sonst hat sich manches zum Guten verändert. Nur sind die Lebenshaltungskosten noch sehr hoch und es langt gerade zum Essen und für die nötigsten Anschaffungen. Zur Zeit stecken wir in einer schweren Krise, denn es gibt eine sehr hohe Zahl von Arbeitslosen und Kurzarbeitern, was sich merklich bemerkbar macht. Als Gewerkschafusfunktionäre haben wir jetzt sehr viel Arbeit, denn es will vielen geholfen sein. Es ist nur gut, daß es wenigstens in der Gewerkschaftsbewegung eine Einheit gibt, obzwar von verschiedenen Seiten der Versuch gemacht wird diese wieder zu zerreißen. Wollen wir aber hoffen, daß dieses nicht gelingt, denn die Zersplitterung innerhalb der Arbeiterschaft politisch gesehen ist genau so arg wie wir es vor 1933 feststellen mußten. Der Wahlkampf zum Bundestag hat dies wieder recht deutlich gezeigt. Gestern haben ja nun die Wahlen stattgefunden, und da hat sich ein merklicher Rechtsruck bemerkbar gemacht, der zeigt, daß die Arbeiterparteien eben nicht in der Lage sind, die Masse der Arbeiterschaft einem großen Ziel entgegenzuführen'. Wenn wir nicht noch mehr eine rückläufige Bewegung machen wollen, müssen in Zukunft neue Wege gefunden werden, nur wird dies ein schwerer Kampf sein bei der Zersplitterung der Arbeiterschaft. Wir können nur hoffen, daß die Ereignisse der Vergangenheit doch noch einen Weg finden lassen, um nicht noch einmal eine gleiche Lage herauf zu beschwören. Leider vermissen wir hierbei die Jugend, welche wir früher hatten. Die Aufgaben sind in Zukunft für uns keine leichten. Ich jedenfalls will versuchen im Rahmen der Gewerkschaft diesem Ziele näherzukommen, denn nur hier ist es bis jetzt möglich gewesen, von einer einheitlichen Bewegung zu sprechen.

Nun habe ich Euch einiges aus unserem Geschehen mitgeteilt und ich glaube, daß auch Ihr rege daran interessiert seid, was noch werden wird. Für heute will ich meine Zeilen beenden in der Hoffnung, daß es Euch allen recht gut gehen möge, und grüße Euch auf das herzlichste

Euer Werner Mager nebst Gattin

\footnotetext{
' Zum Ergebnis der Wahlen zum ersten deutschen Bundestag vgl. Anm. 3 zum Brief Oskar Triebels vom I 2.8. 1949, S. 88 .
} 